\title{
Effect of Different Isoenergetic Breakfast Compositions on Blood Glucose Regulation, Energy Allocation and Satiety
}

\author{
Sadaf Koohkan' ${ }^{1}$, Mana Golsorkhi ${ }^{2}$, Denise Schaffner ${ }^{3}$, Daniel Konig ${ }^{1}$, Peter Deibert ${ }^{2}$, H David \\ McCarthy $^{4}$ and Aloys Berg ${ }^{1 *}$ \\ ${ }^{1}$ Department of Nutrition, Institute of Sport and Sport Science, University of Freiburg, Germany \\ ${ }^{2}$ National Institute for Health Research Clinical Research Network: Diabetes, Metabolic \& Endocrine and Renal Disorders, Imperial College London, UK \\ ${ }^{3}$ Department of Rehabilitative and Praventive Sports Medicine, University Hospital Freiburg, University Hospital Freiburg, Germany \\ ${ }^{4}$ Department of Health and Human Sciences, London Metropolitan University, London, UK
}

Received: August 25, 2014; Accepted: September 06, 2014; Published: September 18, 2014

*Corresponding author: Prof. Aloys Berg, Department of Nutrition, Institute for Sport and Sport Science, University of Freiburg, Schwarzwaldstrasse 175, D-79117 Freiburg, Germany, E-mail: aloys.berg@klinikum.uni-freiburg.de

\begin{abstract}
Background and purpose: The increasing prevalence of overweight and obesity among adults, demands improved dietary strategies for weight management and metabolic competence. Hence, the objective of this study was to assess the short-term effects of breakfasts with varying macronutrient composition on blood glucose regulation, energy metabolism and satiety.
\end{abstract}

Methods: This study examined ten healthy males $(25.6 \pm 4.4$ yrs; BMI $23.2 \pm 0.9 \mathrm{~kg} / \mathrm{m}^{2}$ ) fed isoenergetic breakfasts rich in either Carbohydrate $[\mathrm{CH}]$ (68\% of energy), Fat [Fat] (64\% of energy) or Protein [P] (35\% of energy) or a breakfast which reflected the individuals Normal $[\mathrm{N}]$ breakfast composition. Blood glucose and lactate, resting oxygen consumption $\left(\mathrm{VO}_{2}\right)$, Respiratory Quotient $(\mathrm{RQ})$ and satiety feeling were measured. All breakfasts with the exception of the individual normal breakfast variant were isoenergetic and all contained the same amount of dietary fiber. As a non-dietary control, subjects drank $200 \mathrm{ml}$ water on one test day, with the same metabolic parameters measured.

Results: Compared with the water control day, there was a significant macronutrient-induced change in the metabolic parameters. The most significant increases in blood glucose were found after the Carbohydrate breakfast and the individual normal breakfast, whereas the Fat and Protein-rich breakfasts induced comparatively smaller blood glucose responses. Only the Proteinrich breakfast led to significant increases in resting $\mathrm{VO}_{2}$ (up to $30 \%$ ) without changes in RQ. Finally, the Protein-rich breakfast induced the highest satiety feeling.

Conclusions: Although the Protein-induced effects may initially appear minor, the combination of a reduced glycemic response, increased $\mathrm{VO}_{2}$, a proportionately high fat oxidation and a stronger satiety effect may support the use of this dietary approach for healthy weight management in normal weight men.

Keywords: Macronutrients; Energy Metabolism; Satiety; Weight regulation

\section{Introduction}

An imbalance between energy intake and expenditure drives excess weight gain [1], with a long-term positive balance resulting in overweight and obesity. Numerous factors influence food and energy intake including exogenous factors such as food portion sizes macronutrient composition of the diet, as well as the social environment. Endogenous parameters include feelings of hunger and satiety, eating frequency and the psychology of individuals as well as their level of physical activity [2]. Taken together, these factors can prove to be challenging for individuals wishing to manage their body weight via a diet and lifestyle approach. Nevertheless a strong sensation of meal-induced satiety could be a key determinant of an individual's ability to achieve energy balance over the course of a day and in the longer term. Different foods and macronutrients vary in their satiety-inducing properties although it remains unclear if there is an optimal ratio of macronutrients within a meal to promote the most robust satiety response. Additionally, macronutrients also vary in their ability to increase metabolic rate and to influence post-prandial glycemia; both if which can influence the ability to maintain a healthy body weight. Indeed the latter has also been linked to poorer satiety response due to the concomitant insulin release. During eating, the feeling of satiety should appear well-timed to avoid excessive energy consumption. Ideally this regulatory mechanism should help maintain a healthy body weight in adults, however rising prevalence of obesity and other eating disorders suggest that the satiety mechanism fails or is being over-ridden in many individuals [3]. If an ideal macronutrient composition of a meal could be identified, it could be promoted to encourage people, to follow who wish to maintain a healthy body weight, as well as acting as a new strategy to help reduce body weight and body fatness in those who are already overweight or obese [4-6]. Thus the purpose of this study was to evaluate the shortterm diet-induced effects of different test breakfasts on satiety as well as on blood glucose and lactate response, resting energy expenditure and $\mathrm{RQ}$ and hence fat oxidation over four hours post consumption.

\section{Materials and Methods}

\section{Participants and recruitment}

In this study, ten healthy male volunteers $(25.6 \pm 4.4 \mathrm{yrs}$; BMI, 
$23.2 \pm 0.9 \mathrm{~kg} / \mathrm{m}^{2}$ ) were recruited. All volunteers were within the healthy BMI range, non-smokers, free from known food allergies, metabolic diseases and none regularly used any medication. All volunteers were interviewed and screened before participating in this study at the Department of Sports Medicine of the Freiburg University Hospital.

This study was conducted according to the guidelines laid down in the Declaration of Helsinki, and all procedures involving human subjects were approved by the Ethics Commission of Freiburg University (EK-Freiburg 143/03-110224). All participants started the study after providing written informed consent.

\section{Screening}

The screening involved anthropometric, physical and blood examinations as well as a performance diagnostic. Height was measured to the nearest $0.1 \mathrm{~cm}$ without shoes and body weight was measured utilizing a digital scale. Body Mass Index (BMI) was then calculated. Body density was predicted from skinfold thickness measurement using skinfold calipers (Lange Skinfold Caliper, Beta Technology Inc., Noblesville, USA) at 4 sites (m. biceps brachii, $\mathrm{m}$. triceps brachii, suprailliacal, subscapular) and percentage fat mass was derived using the Siri equation. The performance diagnostic [7] was achieved using standardized and approved methods in the Department of Sports Medicine. Using a lactate analysis, the individual's relative $\mathrm{VO}_{2}$ max was calculated. Subjects' physical characteristics are presented in Table 1.

\section{Study design}

The effects of the participants' responses to the different diets were tested on five separate occasions following an overnight fast. Participants were asked not to consume any food or beverages except water after 11:00 pm the night before test days and refrain from exercise (beyond normal daily activities) and alcohol the day before and on the morning of the test days. Test variables were measured in the morning after fasting. A randomized scheme was used in which baseline and postprandial parameters were measured over a period of four hours after consumption of the test breakfast. The measurements were performed at intervals of at least one week. Each participant was tested on the same day of the week and at the same time of the day. On each test day, one of five breakfast variations was offered. Breakfasts were prepared freshly in the morning. Participants were instructed to eat and drink everything within 10 minutes. Water was allowed ad libitum throughout the test. Participants were assigned a sitting activity in the morning of the test day to avoid additional energy expenditure from physical activity.

\section{Test meal}

Breakfasts were either rich in Carbohydrate [CH] $(68 \%$ of energy), fat [Fat] (64\% of energy) or Protein [P] (35\% of energy) Table 2. All breakfasts were approximately $700 \mathrm{kcal}$ and similar in dietary fibre content ( $6.1 \mathrm{~g}-6.4 \mathrm{~g}$ per meal). Additionally, the composition of each subject's individual habitual or Normal [N] breakfast was imitated and also designed to approximate $700 \mathrm{kcal} / \mathrm{meal}$. As a control, participants received $200 \mathrm{ml}$ water to drink on an additional test day. The compositions of the breakfasts are given in Table 3.

\section{Test parameters}

The state of satiety was recorded at determined time points across the study using a Borg scale [8]. Blood glucose and lactate concentrations were analyzed using an enzymatic amperometric glucose and lactate sensor (EBIO plus, EKF-diagnostic GmbH, Magdeburg). Resting oxygen uptake $\left(\mathrm{VO}_{2}\right)$ and Respiratory Quotient (RQ) were measured by indirect calorimetry using breathing mask with volume sensor of spiroergometry station (ZAN 600 USB CPX, nSpire Health GmbH, Oberthulba).

\section{Blood samples}

Blood samples were collected from the earlobe in $20 \mu$ glass capillaries (EKF-diagnostic GmbH, Magdeburg) after fasting (0 $\min$ ) and 15, 30, 45, 60, 90, 120, 150, 180, 210 and 240 min after consuming the test breakfast.

\section{Respiratory parameters}

Respiratory parameters were measured after fasting ( $0 \mathrm{~min}$ ) and 1, 2, 3 and 4 hours after consuming the test breakfast by continuous flow indirect calorimetry. Oxygen uptake $\left(\mathrm{VO}_{2}\right)$ and carbon dioxide $\left(\mathrm{VCO}_{2}\right)$ were recorded continuously breath by breath and the Respiratory Quotient (RQ) calculated as the ratio of $\mathrm{VCO}_{2}$ to $\mathrm{VO}_{2}$

\section{Record of satiety}

Participants recorded their feeling satiety after fasting $(0$ $\min$ ) and $20 \mathrm{~min}$ and 1, 2, 3 and 4 hours after eating. The feeling was rated using a scale with $0=$ no hunger, $2=$ slight hunger, $4=$ moderate hunger, $6=$ strong hunger, $8=$ very strong hunger, $10=$ strongest imaginable hunger [8]

\section{Statistical analyses}

For each participant, complete datasets were available in Microsoft ${ }^{\circledR}$ Excel XP spreadsheets. The results are expressed as means \pm standard deviations for all parameters. Incremental Areas Under the Curve (IAUCs) were established for the parameters tested for each subject. The IAUC was defined as the difference between the integrated area under the curve of the postprandial response and the rectangular area determined by the associated fasting value. Thereby, positive and negative areas were included. For satiety, the sum of postprandial satiety scale values ( $\Sigma 20240 \mathrm{~min}$ ) instead of IAUCs was calculated. Each of the four test breakfasts was compared with the control (water). In addition, the variance between diets in terms of the three main nutrients (Carbohydrate, Fat and Protein) was analyzed. All statistical significances were calculated using a combined test (Friedman- and Wilcoxon-Test) with Bonferroni-Holm adjustment for multiple comparisons [9]. Statistical significance was defined as $p<0.05$. All analyses were performed using SPSS 18.02 .

\section{Results}

\section{The satiety}

Reported feeling of satiety significantly increased following 
all breakfast types, with the exception of the (water) control. After correcting for multiple comparisons, all breakfast variations initiated significant changes in satiety or, in the case of the control, in hunger feeling (Table 4). Compared with the control breakfast, satiety feeling 20 min postprandially was significantly enhanced; reported satiety slowly decreased through the course of the testing morning. Participants reported no appreciable differences between the four breakfasts in satiety at the end of the test period ( $240 \mathrm{~min}$ postprandially). The sum of the satiety scale values ( $\Sigma 20-240 \mathrm{~min}$ ) (Table 4 ), followed the order $\mathrm{P}>\mathrm{CH}$ $=\mathrm{N}>$ Fat. The control (water) meal induced twice the feeling of hunger compared to the test breakfasts. Comparing the sum of satiety scale values for the three main nutrients, significant differences in satiety feeling was identified only without correcting for multiple comparisons: the Protein-rich breakfast variant caused a significantly higher satiety feeling compared with the Carbohydrate and Fat-rich breakfasts.

\section{Blood glucose}

All breakfast variations except for the control induced a significant postprandial blood glucose peak $(p \leq 0.05)$. However, after correction for multiple comparisons, these increases compared to the control were no longer significant. At the postprandial peak at $30 \mathrm{~min}$ when the absolute values reached their maximum, the Carbohydrate-rich breakfast induced a 56\% increase (the largest) and the Protein-rich breakfast induced a $23 \%$ increase (the smallest) in blood glucose compared to the control (Table 5). IAUCs (Figure 1) showed the following order: $\mathrm{CH}>\mathrm{N}>$ Fat $\approx \mathrm{P}$. Comparing the IAUCs of the three main nutrients (Carbohydrate, Fat and Protein), the glucose increase after the Carbohydrate-rich breakfast significantly differed from the IAUCs of the Fat and the Protein-rich breakfast variants.

\section{Blood lactate}

All breakfast variations except for the control resulted in a significant lactate peak $(p \leq 0.05)$. However, after correction for multiple comparisons, only the lactate increases following the Carbohydrate and the Protein-rich breakfasts remained significant (Table 5). The highest absolute lactate values were achieved after 45-60 min. In contrast to blood glucose, the lactate concentration fell below the associated fasting lactate value at the end of the tests. For the postprandial maximal absolute lactate value, the Carbohydrate-rich test breakfast induced a $184 \%$ increase (the largest) in blood lactate level, whereas the Fat-rich breakfast resulted in the smallest increase. IAUCs (Figure 1) were in the following order: $\mathrm{CH}>\mathrm{N} \approx \mathrm{P}>$ Fat

$\mathrm{VO}_{2}$

Compared with the water control, postprandial $\mathrm{VO}_{2}$ significantly increased following all breakfast types. After correction for multiple comparisons, all breakfast types resulted

Table 1: Means \pm SD of the characteristics of the subjects $(n=10)$ enrolled.

\begin{tabular}{|c|c|c|c|c|c|c|}
\hline & $\begin{array}{c}\text { Age } \\
{[\mathrm{y}]}\end{array}$ & Weight $[\mathrm{kg}]$ & Height $[\mathrm{cm}]$ & $\begin{array}{c}\text { BMI } \\
{\left[\mathrm{kg} / \mathrm{m}^{2}\right]}\end{array}$ & $\begin{array}{c}\text { Fat mass } \\
{[\%]}\end{array}$ & $\mathbf{V 0 2}$ max $_{\text {max }}[\mathrm{ml} / \mathrm{kg} / \mathrm{min}]$ \\
\hline $\begin{array}{c}\text { Mean } \pm \text { SD } \\
(\mathbf{n = 1 0}\end{array}$ & $25.6 \pm 4.4$ & $78.4 \pm 5.1$ & $184.0 \pm 6.6$ & $23.2 \pm 0.9$ & $17.6 \pm 3.0$ & $55.9 \pm 4.1$ \\
\hline
\end{tabular}

Table 2: Energy composition of the five breakfast variations.

\begin{tabular}{|c|c|c|c|c|c|}
\hline & $\begin{array}{c}\text { CH } \\
\text { [\% of energy] }\end{array}$ & $\begin{array}{c}\text { Fat } \\
\text { [\% of energy] }\end{array}$ & $\begin{array}{c}\text { P } \\
\text { [\% of energy] }\end{array}$ & $\begin{array}{c}\text { Dietary fiber } \\
\text { [g] }\end{array}$ & $\begin{array}{c}\text { Energy } \\
\text { [kcal] }\end{array}$ \\
\hline CH-rich breakfast & 68 & 23 & 9 & 6.4 & 715 \\
\hline Fat-rich breakfast & 26 & 64 & 10 & 6.1 & 688 \\
\hline P-rich breakfast & 33 & 32 & 35 & 6.4 & 700 \\
\hline $\begin{array}{c}\text { Individual normal } \\
\text { breakfast (Mean }\end{array}$ & 56 & $31-10$ & 13 & 0 & 700 \\
\hline Water breakfast (control) & 0 & 0 & 0 & 0 & 0 \\
\hline
\end{tabular}

Data were calculated with the nutrition software DGE-PC Professional)

Table 3: Composition and Total Weight [TW] of the five breakfast variations.

\begin{tabular}{|c|c|c|}
\hline & Composition/Ingredients & TW $[g]$ \\
\hline CH-rich breakfast & $\begin{array}{l}130 \text { g baguette, } 10 \mathrm{~g} \text { butter, } 10 \mathrm{~g} \text { jam, } 10 \text { g nut-nougat-cream (sweet), } \\
150 \text { g yoghurt ( } 3.8 \% \text { ) with fruit preparation, } 200 \mathrm{ml} \text { orange juice }\end{array}$ & 510 \\
\hline Fat-rich breakfast & $\begin{array}{l}65 \text { g croissant (flaky pastry), } 30 \text { g pumpernickel, } 20 \text { g butter, } 20 \text { g cheese, } \\
15 \text { g salami, } 15 \text { g salmon (cured), } 50 \text { g pepper (pepper), } 200 \mathrm{ml} \text { water or tea }\end{array}$ & 415 \\
\hline P-rich breakfast & $\begin{array}{l}35 \mathrm{~g} \text { multi-grain bread, } 25 \mathrm{~g} \text { whole-grain bread, } 6 \mathrm{~g} \text { butter, } 50 \mathrm{~g} \text { trout } \\
\text { (cured), } 10 \mathrm{~g} \text { honey, } 50 \mathrm{~g} \text { curd cheese (low-fat), } 5 \mathrm{~g} \text { nuts (fresh), } 80 \mathrm{~g} \\
\text { orange (fresh, peeled), } 70 \mathrm{~g} \text { hen's egg (hard-cooked), } 50 \mathrm{~g} \text { soy-based } \\
\text { protein-powder (Almased (囚) dissolved in } 200 \mathrm{ml} \text { water }\end{array}$ & 581 \\
\hline $\begin{array}{l}\text { Individual normal } \\
\text { breakfasts }\end{array}$ & $\begin{array}{c}\text { Not listed } \\
\text { Mean }_{1-10}\end{array}$ & 507 \\
\hline Water breakfast (control) & $200 \mathrm{ml}$ water & 200 \\
\hline
\end{tabular}


Table 4: Effects of different breakfast variations on satiety feeling according to time.

\begin{tabular}{|c|c|c|c|c|c|c|c|}
\hline \multicolumn{7}{|c|}{ Satiety Scale (Mean \pm SD) $(n=10)$} & \multirow[b]{2}{*}{$\Sigma_{20-240[\min ]}$} \\
\hline & 0 [min] & 20 [min] & $60[\mathrm{~min}]$ & $120[\mathrm{~min}]$ & 180 [min] & 240 [min] & \\
\hline CH-rich breakfast & $4.8 \pm 1.4$ & $0.6^{\mathrm{a}} \pm 0.7$ & $0.9^{\mathrm{a}} \pm 1.0$ & $1.8^{\mathrm{a}} \pm 1.1$ & $2.5^{\mathrm{a}} \pm 1.3$ & $3.6^{\mathrm{a}} \pm 1.5$ & 9.32 \\
\hline $\begin{array}{c}\text { Fat-rich } \\
\text { breakfast }\end{array}$ & $4.6 \pm 1.4$ & $1.6^{\mathrm{a}} \pm 1.3$ & $1.9^{\mathrm{a}} \pm 1.2$ & $2.2^{\mathrm{a}} \pm 1.4$ & $2.8^{\mathrm{a}} \pm 2.0$ & $3.8^{\mathrm{ns}} \pm 1.8$ & 12.41 \\
\hline P-rich breakfast & $4.6 \pm 1.8$ & $0.5^{\mathrm{a}} \pm 0.7$ & $0.4^{\mathrm{a}} \pm 0.7$ & $1.0^{\mathrm{a}} \pm 1.0$ & $1.5^{\mathrm{a}} \pm 1.3$ & $3.1^{\mathrm{a}} \pm 1.3$ & 6.55 \\
\hline $\begin{array}{c}\text { Individual } \\
\text { normal } \\
\text { breakfasts }\end{array}$ & $4.6 \pm 2.0$ & $0.9^{\mathrm{a}} \pm 1.0$ & $1.0^{\mathrm{a}} \pm 1.0$ & $1.6^{\mathrm{a}} \pm 1.5$ & $2.5^{\mathrm{a}} \pm 1.5$ & $3.3^{\mathrm{ns}} \pm 1.6$ & 9.28 \\
\hline $\begin{array}{l}\text { Water breakfast } \\
\text { (control) }\end{array}$ & $3.7 \pm 2.1$ & $4.1^{\mathrm{ns}} \pm 1.8$ & $5.0^{\mathrm{a}} \pm 1.8$ & $5.3^{\mathrm{a}} \pm 1.6$ & $5.7^{\mathrm{a}} \pm 1.3$ & $6.7^{\mathrm{a}} \pm 1.5$ & 26.79 \\
\hline
\end{tabular}

After determining a fasting value ( 0 [min]), further measurements were taken after subjects consumed the test breakfasts to determine postprandial satiety feeling over the next four hours. Means and associated Standard Deviations [SD] were calculated from the data of the study subjects ( $=10)$. Data were registered by a questionnaire using the scale with $0=$ no hunger, $2=$ slight hunger, $4=$ moderate hunger, $6=$ strong hunger, $8=$ very strong hunger, $10=$ strongest imaginable hunger. Significances are corrected for multiple comparisons and denoted via letters: ns: non-significant; $\mathbf{a}:{ }^{*} p \leq$ 0.05 vs. the corresponding fasting value

Table 5: Effects of different breakfast variations on blood glucose and blood lactate concentrations over time.

\begin{tabular}{|c|c|c|c|c|c|c|c|c|c|c|c|}
\hline \multicolumn{12}{|c|}{ Lactate $[\mathrm{mg} / \mathrm{dl}]($ Mean \pm SD) $(\mathrm{n}=10)$} \\
\hline & $\mathbf{0}[\mathrm{min}]$ & 15 [min] & 30 [min] & $45[\mathrm{~min}]$ & $60[\mathrm{~min}]$ & 90 [min] & 120 [min] & ] 150 [min] & ] $180[\mathrm{~min}]$ & ] 210 [min] & $240 \mathrm{~min}$ \\
\hline $\begin{array}{c}\text { CH-rich } \\
\text { breakfast }\end{array}$ & $\begin{array}{c}0.89 \pm \\
0.35\end{array}$ & $\begin{array}{c}1.06^{\mathrm{ns}} \pm \\
0.35\end{array}$ & $\begin{array}{c}1.75^{\mathrm{a}} \pm \\
0.34\end{array}$ & $\begin{array}{c}1.93^{\mathrm{a}} \pm \\
0.39\end{array}$ & $\begin{array}{c}1.68^{\mathrm{a}} \pm \\
0.49\end{array}$ & $\begin{array}{c}1.45^{\mathrm{a}} \pm \\
0.43\end{array}$ & $\begin{array}{c}1.17^{\text {ns }} \pm \\
0.31\end{array}$ & $\begin{array}{c}0.92^{\mathrm{ns}} \pm \\
0.21\end{array}$ & $\begin{array}{c}0.85^{\mathrm{ns}} \pm \\
0.14\end{array}$ & $\begin{array}{c}0.80^{\text {ns }} \pm \\
0.17\end{array}$ & $\begin{array}{c}0.72^{\text {ns }} \pm \\
0.12\end{array}$ \\
\hline $\begin{array}{c}\text { Fat-rich } \\
\text { breakfast }\end{array}$ & $\begin{array}{c}0.85 \pm \\
0.30\end{array}$ & $\begin{array}{c}0.77^{\text {ns }} \pm \\
0.24\end{array}$ & $\begin{array}{c}0.88^{\text {ns }} \pm \\
0.24\end{array}$ & $\begin{array}{c}0.91^{\mathrm{ns}} \pm \\
0.26\end{array}$ & $\begin{array}{c}0.94^{\text {ns }} \pm \\
0.24\end{array}$ & $\begin{array}{c}0.86^{\mathrm{ns}} \pm \\
0.16\end{array}$ & $\begin{array}{c}0.73^{\text {ns }} \pm \\
0.15\end{array}$ & $\begin{array}{c}0.69^{\text {ns }} \pm \\
0.16\end{array}$ & $\begin{array}{c}0.65^{\mathrm{ns}} \pm \\
0.10\end{array}$ & $\begin{array}{c}0.61^{\text {ns }} \pm \\
0.11\end{array}$ & $\begin{array}{c}0.58^{\text {ns }} \pm \\
0.08\end{array}$ \\
\hline $\begin{array}{c}\text { P-rich } \\
\text { breakfast }\end{array}$ & $\begin{array}{c}0.83 \pm \\
0.22\end{array}$ & $\begin{array}{c}0.83^{\text {ns }} \pm \\
0.26\end{array}$ & $\begin{array}{l}1.11^{\mathrm{a}} \pm \\
0.27\end{array}$ & $\begin{array}{l}1.21^{\mathrm{ns}} \pm \\
0.24\end{array}$ & $\begin{array}{l}1.20^{\text {ns }} \pm \\
0.25\end{array}$ & $\begin{array}{c}1.01^{\mathrm{ns}} \pm \\
0.14\end{array}$ & $\begin{array}{l}0.91^{\mathrm{ns}} \pm \\
0.13\end{array}$ & $\begin{array}{l}0.78^{\text {ns }} \pm \\
0.15\end{array}$ & $\begin{array}{l}0.77^{\mathrm{ns}} \pm \\
0.17\end{array}$ & $\begin{array}{c}0.72^{\mathrm{ns}} \pm \\
0.14\end{array}$ & $\begin{array}{c}0.62^{\mathrm{ns}} \pm \\
0.15\end{array}$ \\
\hline $\begin{array}{c}\text { Individ. normal } \\
\text { breakfasts }\end{array}$ & $\begin{array}{l}0.91 \pm \\
0.27\end{array}$ & $\begin{array}{l}0.87^{\text {ns }} \pm \\
0.32\end{array}$ & $\begin{array}{c}1.22^{\text {ns }} \pm \\
0.39\end{array}$ & $\begin{array}{l}1.41^{\text {ns }} \pm \\
0.50\end{array}$ & $\begin{array}{l}1.31^{\text {ns }} \pm \\
0.36\end{array}$ & $\begin{array}{c}1.15^{\text {ns }} \pm \\
0.34\end{array}$ & $\begin{array}{l}0.91^{\text {ns }} \pm \\
0.23\end{array}$ & $\begin{array}{l}0.87^{\text {ns }} \pm \\
0.16\end{array}$ & $\begin{array}{c}0.79^{\text {ns }} \pm \\
0.14\end{array}$ & $\begin{array}{l}0.74^{\mathrm{ns}} \pm \\
0.13\end{array}$ & $\begin{array}{c}0.69^{\text {ns }} \pm \\
0.11\end{array}$ \\
\hline $\begin{array}{c}\text { Water } \\
\text { breakfast } \\
\text { (control) }\end{array}$ & $\begin{array}{c}0.82 \pm \\
0.27\end{array}$ & $\begin{array}{c}0.67^{\mathrm{ns}} \pm \\
0.22\end{array}$ & $\begin{array}{c}0.67^{\mathrm{ns}} \pm \\
0.20\end{array}$ & $\begin{array}{c}0.68^{\text {ns }} \pm \\
0.16\end{array}$ & $\begin{array}{c}0.66^{\text {ns }} \pm \\
0.15\end{array}$ & $\begin{array}{c}0.68^{\mathrm{ns}} \pm \\
0.17\end{array}$ & $\begin{array}{c}0.66^{\mathrm{ns}} \pm \\
0.17\end{array}$ & $\begin{array}{c}0.66^{\mathrm{ns}} \pm \\
0.14\end{array}$ & $\begin{array}{l}0.68^{\mathrm{ns}} \pm \\
0.13\end{array}$ & $\begin{array}{l}0.70^{\text {ns }} \pm \\
0.16\end{array}$ & $\begin{array}{l}0.58^{\mathrm{ns}} \pm \\
0.09\end{array}$ \\
\hline \multicolumn{12}{|c|}{ Glucose $[\mathrm{mg} / \mathrm{dl}]($ Mean \pm SD) $(\mathrm{n}=10)$} \\
\hline & 0 [min] & 15 [min] & 30 [min] & 45 [min] & 60 [min] & 90 [min] & $120[\mathrm{~min}]$ & 150 [min] & 180 [min] & 210 [min] & $240 \mathrm{~min}]$ \\
\hline $\begin{array}{c}\text { CH-rich } \\
\text { breakfast }\end{array}$ & $73 \pm 5$ & $93^{a} \pm 12$ & $122^{\mathrm{a}} \pm 19$ & $105^{\mathrm{a}} \pm 15$ & $87^{a} \pm 13$ & $91^{\mathrm{a}} \pm 9$ & $88^{a} \pm 6$ & $90^{\mathrm{a}} \pm 7$ & $81^{\text {ns }} \pm 9$ & $79^{\mathrm{ns}} \pm 6$ & $76^{\mathrm{ns}} \pm 7$ \\
\hline $\begin{array}{c}\text { Fat-rich } \\
\text { breakfast }\end{array}$ & $76 \pm 5$ & $87^{a} \pm 9$ & $101^{\mathrm{a}} \pm 12$ & $93^{\mathrm{a}} \pm 11$ & $82^{\mathrm{ns}} \pm 13$ & $80^{\mathrm{ns}} \pm 9$ & $81^{\mathrm{ns}} \pm 6$ & $80^{\mathrm{ns}} \pm 3$ & $80^{\text {ns }} \pm 3$ & $79^{\text {ns }} \pm 4$ & $80^{\mathrm{ns}} \pm 5$ \\
\hline $\begin{array}{c}\text { P-rich } \\
\text { breakfast }\end{array}$ & $77 \pm 6$ & $85^{\mathrm{a}} \pm 7$ & $96^{\mathrm{a}} \pm 8$ & $85^{\mathrm{ns}} \pm 8$ & $79^{\mathrm{ns}} \pm 8$ & $82^{\mathrm{ns}} \pm 8$ & $82^{\mathrm{ns}} \pm 6$ & $85^{\mathrm{ns}} \pm 7$ & $83^{\text {ns }} \pm 4$ & $82^{\mathrm{ns}} \pm 7$ & $82^{\mathrm{ns}} \pm 6$ \\
\hline $\begin{array}{c}\text { Individ. } \\
\text { normal } \\
\text { breakfasts }\end{array}$ & $72 \pm 5$ & $85^{\mathrm{a}} \pm 9$ & $112^{\mathrm{a}} \pm 19$ & $104^{\mathrm{ns}} \pm 22$ & $84^{\mathrm{ns}} \pm 11$ & $85^{\mathrm{ns}} \pm 9$ & $83^{\text {ns }} \pm 10$ & $83^{\mathrm{ns}} \pm 14$ & $81^{\mathrm{ns}} \pm 10$ & $78^{\mathrm{ns}} \pm 7$ & $76^{\mathrm{ns}} \pm 8$ \\
\hline $\begin{array}{c}\text { Water } \\
\text { breakfast } \\
\text { (control) }\end{array}$ & $75 \pm 7$ & $78^{\mathrm{ns}} \pm 8$ & $78^{\mathrm{ns}} \pm 6$ & $78^{\mathrm{ns}} \pm 6$ & $78^{\mathrm{ns}} \pm 7$ & $76^{\mathrm{ns}} \pm 6$ & $77^{\mathrm{ns}} \pm 7$ & $76^{\mathrm{ns}} \pm 6$ & $77^{\mathrm{ns}} \pm 6$ & $75^{\mathrm{ns}} \pm 6$ & $77^{\mathrm{ns}} \pm 6$ \\
\hline
\end{tabular}

After determining a fasting value (0 [min]), further blood samples were taken after subjects consumed the test breakfasts to determine postprandial values over the next four hours. Means and associated Standard Deviations [SD] were calculated from data of the study subjects $(\mathrm{n}=10)$.

in significant augmentations in $\mathrm{VO}_{2}$ (Table 6). Peak $\mathrm{VO}_{2}$ values occurred 60-120 min after breakfast and returned to fasting levels 180-240 min postprandially. Only the Protein-rich breakfast sustained a significant rise in $\mathrm{VO}_{2}$ over the entire 240 min period (Table 6). A mean peak oxygen consumption of 0.35 $\mathrm{l} / \mathrm{min}$ was achieved following the Carbohydrate and Protein-rich breakfasts. However, the decline was more pronounced in the
Carbohydrate-rich breakfast, such that the Protein-rich breakfast induced a slightly higher total $\mathrm{VO}_{2}$ increase over 4 hours. IAUCs were in the following sequence: $\mathrm{P} \approx \mathrm{CH}>\mathrm{N}>$ Fat. For the control, the IAUC was close to zero (Figure 2).

\section{Respiratory Quotient (RQ)}

Respiratory Quotient (RQ) was also influenced by breakfast 
Table 6: Effects of different breakfast variations on oxygen consumption [VO2] and the Respiratory Quotient [RQ] according to time.

\begin{tabular}{|c|c|c|c|c|c|}
\hline \multicolumn{5}{|c|}{$\operatorname{V02}[1 / \mathrm{min}]($ Mean $\pm \operatorname{SD})(\mathrm{n}=10)$} & \multirow[b]{2}{*}{$240[\mathrm{~min}]$} \\
\hline & 0 [min] & $60[\mathrm{~min}]$ & $120[\mathrm{~min}]$ & $180[\mathrm{~min}]$ & \\
\hline CH-rich breakfast & $0.27 \pm 0.05$ & $0.35^{\mathrm{a}} \pm 0.05$ & $0.35^{\mathrm{a}} \pm 0.06$ & $0.31^{\mathrm{a}} \pm 0.04$ & $0.29^{\text {ns }} \pm 0.03$ \\
\hline Fat-rich breakfast & $0.28 \pm 0.04$ & $0.31^{\mathrm{a}} \pm 0.04$ & $0.31^{\mathrm{a}} \pm 0.04$ & $0.29^{\text {ns }} \pm 0.03$ & $0.28^{\mathrm{ns}} \pm 0.03$ \\
\hline P-rich breakfast & $0.27 \pm 0.03$ & $0.34^{\mathrm{a}} \pm 0.03$ & $0.35^{\mathrm{a}} \pm 0.04$ & $0.33^{\mathrm{a}} \pm 0.03$ & $0.30^{\mathrm{a}} \pm 0.03$ \\
\hline $\begin{array}{c}\text { Individual normal } \\
\text { breakfasts }\end{array}$ & $0.27 \pm 0.04$ & $0.32^{\mathrm{a}} \pm 0.05$ & $0.32^{\mathrm{a}} \pm 0.04$ & $0.31^{\text {ns }} \pm 0.04$ & $0.28^{\mathrm{ns}} \pm 0.04$ \\
\hline $\begin{array}{l}\text { Water breakfast } \\
\text { (control) }\end{array}$ & $0.28 \pm 0.05$ & $0.28^{\mathrm{ns}} \pm 0.05$ & $0.28^{\text {ns }} \pm 0.04$ & $0.27^{\mathrm{ns}} \pm 0.03$ & $0.28^{\mathrm{ns}} \pm 0.02$ \\
\hline \multicolumn{5}{|c|}{ RQ $($ Mean $\pm S D)(n=10)$} & \\
\hline & 0 [min] & 60 [min] & $120[\mathrm{~min}]$ & $180[\mathrm{~min}]$ & $240[\mathrm{~min}]$ \\
\hline CH-rich breakfast & $0.86 \pm 0.03$ & $0.93^{\mathrm{a}} \pm 0.03$ & $0.92^{\mathrm{a}} \pm 0.03$ & $0.91^{\mathrm{a}} \pm 0.03$ & $0.86^{\text {ns }} \pm 0.03$ \\
\hline Fat-rich breakfast & $0.87 \pm 0.03$ & $0.84^{\mathrm{ns}} \pm 0.04$ & $0.84^{\mathrm{ns}} \pm 0.04$ & $0.83^{\text {ns }} \pm 0.05$ & $0.82^{\mathrm{a}} \pm 0.04$ \\
\hline P-rich breakfast & $0.85 \pm 0.04$ & $0.85^{\mathrm{ns}} \pm 0.03$ & $0.85^{\mathrm{ns}} \pm 0.04$ & $0.86^{\mathrm{ns}} \pm 0.03$ & $0.86^{\mathrm{ns}} \pm 0.05$ \\
\hline $\begin{array}{c}\text { Individual normal } \\
\text { breakfasts }\end{array}$ & $0.87 \pm 0.04$ & $0.90^{\mathrm{ns}} \pm 0.04$ & $0.90^{\mathrm{ns}} \pm 0.03$ & $0.88^{\text {ns }} \pm 0.03$ & $0.88^{\text {ns }} \pm 0.05$ \\
\hline $\begin{array}{l}\text { Water breakfast } \\
\text { (control) }\end{array}$ & $0.84 \pm 0.03$ & $0.85^{\mathrm{ns}} \pm 0.03$ & $0.83^{\text {ns }} \pm 0.03$ & $0.83^{\text {ns }} \pm 0.04$ & $0.82^{\text {ns }} \pm 0.05$ \\
\hline
\end{tabular}

After determining a fasting value (0 [min]), further measurements were taken after subjects consumed the test breakfasts to determine postprandial values over the next four hours. Means and associated Standard Deviations [SD] were calculated from the data of the study subjects ( $\mathrm{n}=10$ ). Parameters were recorded by spiroergometry. Significances are corrected for multiple comparisons and denoted via letters: ns: non-significant; $\mathbf{a}:{ }^{*} p \leq 0.05$ vs. the corresponding fasting value.

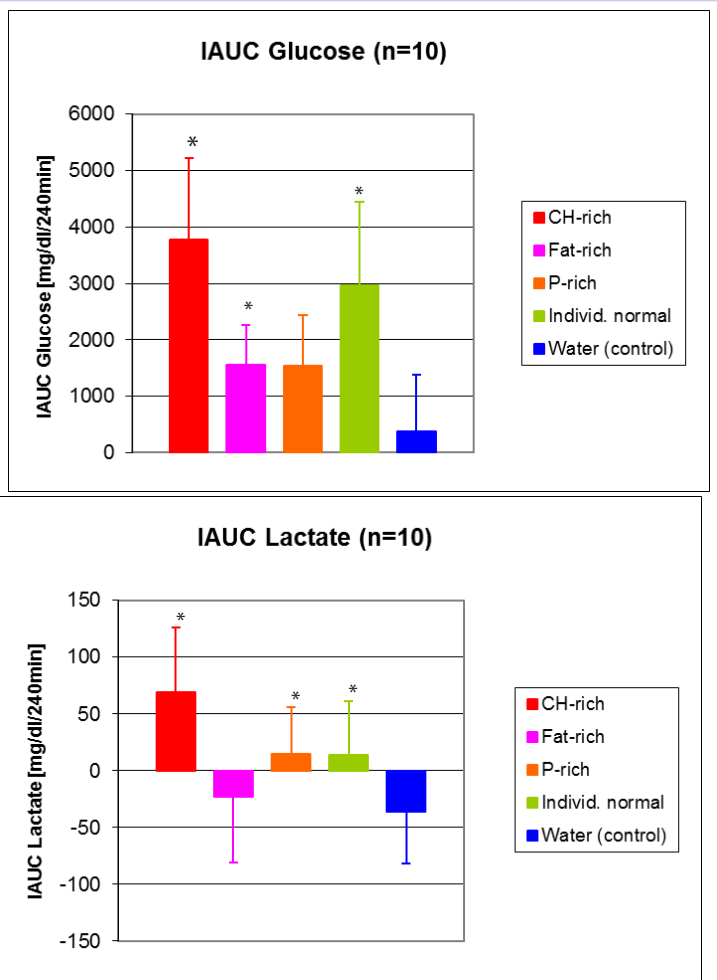

Figure 1: Effects of different breakfast variations on IAUC for blood glucose and lactate. IAUCs were estimated over a time span of four hours after subjects consumed the test breakfast. Illustrated are means and associated standard deviations $[\mathrm{SD}]$ calculated from the data of the study subjects $(\mathrm{n}=10)$. Significances are corrected for multiple comparisons and denoted with ${ }^{*} p \leq 0.05$ vs. control
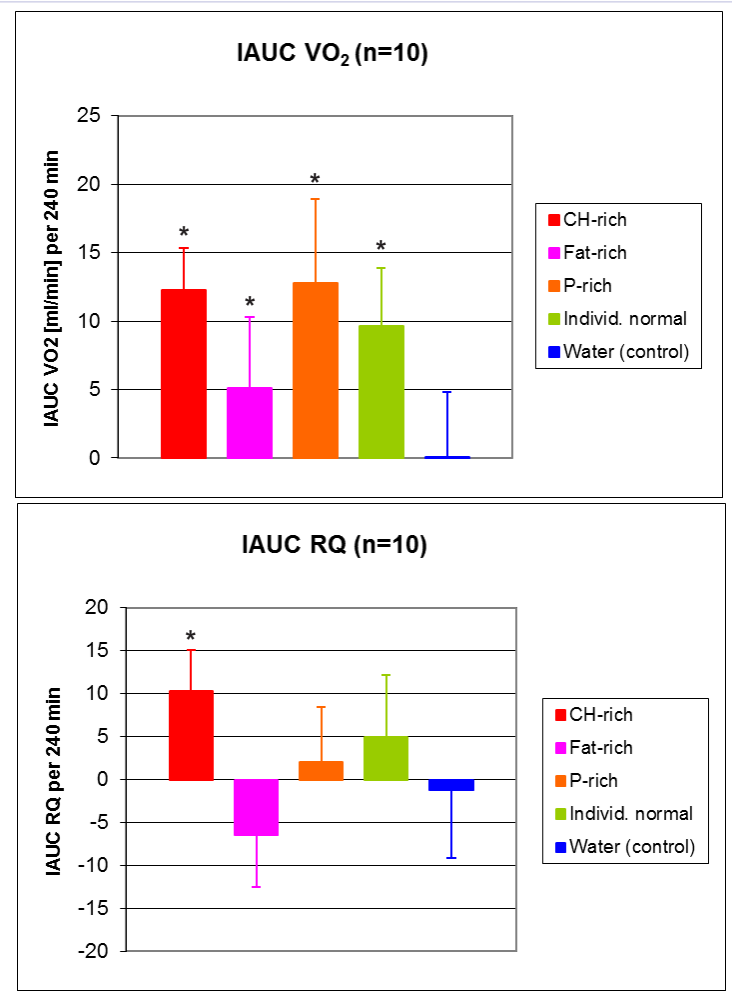

Figure 2: Effects of different breakfast variations on IAUC for oxygen consumption $\left[\mathrm{VO}_{2}\right]$ and the Respiratory Quotient [RQ]. IAUCs were estimated over a time span of four hours after subjects consumed the test breakfast. Illustrated are means and associated Standard Deviations [SD] calculated from data of the study subjects $(\mathrm{n}=10)$. Significances are corrected for multiple comparisons and denoted with ${ }^{*} \mathrm{p} \leq 0.05$ vs. control 
type (Table 5). A significant RQ increase was observed after the Carbohydrate-rich and the individual normal breakfasts. In contrast, the Fat-rich breakfast resulted in a significant decline in RQ. The Protein-rich breakfast and the control (water) showed no significant changes from their fasting values. After correction for multiple comparisons, the RQ increase following the Carbohydrate-rich breakfast and the decrease following the Fat-rich breakfast remained significant (Table 5). The Carbohydrate-rich breakfast induced the maximum RQ rise 60 min postprandially (53\%) compared to the control. IAUCs show a clear order: $\mathrm{CH}>\mathrm{N}>\mathrm{P}>$ Fat. The IAUC for the control was close to zero. If IAUCs of the main nutrients are compared, the Carbohydrate-rich breakfast induced a significantly greater RQ increase compared with the Fat and Protein-rich breakfasts. Additionally, the RQ decline following the Fat-rich test breakfast significantly differed from the small RQ increase after the Proteinrich test breakfast (Figure 2).

\section{Discussion}

This study set out to compare a range of breakfasts rich in either carbohydrate, Fat or Protein on feelings of satiety and a number of metabolic parameters. The intention was to consider whether an optimal level of one particular macronutrient could be proposed as a means of best promoting healthy weight management. The study found that a breakfast high in Protein prolonged satiety promoted an increased in resting metabolic rate and favourably affected postprandial glycaemia compared with the breakfast rich in carbohydrate or Fat.

Several studies have indicated that eating breakfast is a health-promoting behaviour [10-14], even though it may contribute to higher total daily energy intake [15]. In contrast, skipping breakfast is associated with a low frequency of healthpromoting behaviours $[16,17]$. In this study, it could be viewed that the effects of skipping breakfast were imitated in the control breakfast where only plain water was provided. It was found that postprandial values of metabolic and respiratory parameters from the control remained around fasting values; thus, an increase in resting metabolic rate failed to occur. However, at the end of the test day, participants perceived a strong hunger feeling, which could lead to overeating at lunch. In contrast, the four isoenergetic $(\sim 700 \mathrm{kcal})$ breakfast variants yielded clear postprandial responses and macronutrient-dependent effects.

As increased glucose levels promote insulin secretion, inhibit fat oxidation and possibly increase hunger [18,19], an excessive postprandial glucose increase may be unfavourable for healthy weight management [20]. As expected, the high- $\mathrm{CH}$ breakfast variants (the Carbohydrate-rich and the individual normal breakfast) led to the greatest increases in blood glucose. The Fat and Protein-rich test breakfasts resulted in increases in glucose levels that were approximately half that of the individuals usual or normal breakfast. The difference may be explained by a higher fructose content in the Carbohydrate-rich breakfast because more lactate is produced when more fructose is consumed [21]. Variation in postprandial energy expenditure might play an important role in the development of overweight. Indeed, several parameters have been found to be predictive for weight gain including a low basal or resting metabolic rate, high fasting and/or postprandial $\mathrm{RQ}$ which reflect a low fat oxidation rate and low endurance fitness and low spontaneous physical activity [22-25]. With this study, we attempted to make more precise statements about the effects of different breakfast types on energy metabolism. The results indicated that a higher intake of carbohydrate induced a pronounced increase of $\mathrm{VO}_{2}$ but also significantly augmented RQ, indicating an inhibition of fat oxidation. Therefore, it could be suggested that a high carbohydrate breakfast has disadvantageous effects on both blood and respiratory parameters, compared with, for example a high Protein breakfast. The findings also suggest that in relation to long-term weight management, the participants' individual choices for their daily breakfast, which on average, amounted to approximately $56 \%$ of energy, should be questioned. Following the Fat-rich breakfast, the enhancement of $\mathrm{VO}_{2}$ was the lowest, and the postprandial fat oxidation was the highest. Nevertheless less than half the amount of fat consumed was oxidized. It is likely that endogenous fat stores would not be affected by the increased fat oxidation but rather are expanded by the residual dietary fat that was not oxidized. From these findings it could be claimed that the high fat intake cannot be balanced without additional energy expenditure through physical activity. Only the Protein-rich test breakfast was able to influence both respiratory parameters in a direction considered favourable for weight management $\mathrm{VO}_{2}$ increased, RQ was kept constant and postprandial fat oxidation remained at a proportionately high level. For the Protein-rich breakfast, the calculated mean ratio of fat oxidation was $46 \%$ instead of the $32.5 \%$ after the Carbohydrate-rich breakfast.

The results of previous studies indicated that dietary Protein induces a higher and longer thermic effect compared with either carbohydrate or Fat [26-29]. This response may be a key factor which explains why increased dietary Protein seems to prevent weight gain and/or regain after weight loss [30-36]. The fact that there is no capacity to store Protein, and it thus has to be immediately metabolized, may be one explanation for the macronutrient-dependent differences in the thermic effect. The diet-induced increase in $\mathrm{VO}_{2}$ between the Protein and the Carbohydrate-rich breakfasts showed minimal differences (Figure 2). However, by multiplying the amount of additional $\mathrm{VO}_{2}$ with the energy-equivalent for $\mathrm{O}_{2}$ (rounded to $5 \mathrm{kcal} / \mathrm{l} \mathrm{O}_{2}$ ), it was calculated that the consumption of both breakfast variants enhanced EE by approximately $63 \mathrm{kcal}$ during the testing morning. This result is consistent with $9 \%$ of total energy consumed (700 kcal) but in itself does not explain the higher thermic effect after Protein intake. One possible explanation may be the unequal content of the "main" macronutrient of the particular breakfasts. In this study the carbohydrate content of the Carbohydrate-rich breakfast accounted for $68 \%$ of energy, whereas the Protein content of the Protein-rich breakfast accounted for 35\% of energy - approximately half as much. Therefore, the "real" thermic effect of Protein is likely underrepresented. Additionally a longer lasting thermic effect, up to more than six hours after Protein intake could also explain the differences as the increase of $\mathrm{VO}_{2}$ after the Protein-rich breakfast may be underestimated due to a measurement period of only four hours [27,37]. Possibly 
any differences were too small to define a clear macronutrientdependent order for $\mathrm{VO}_{2}$. However, the results of this study provide evidence for an order for $\mathrm{RQ}$ and substrate oxidation: the higher the carbohydrate content and/or the lower the fat content of the breakfast, the higher the postprandial RQ and thus, carbohydrate oxidation, and the lower the postprandial fat oxidation.

Macronutrients are also responsible for satiety.Carbohydrates and especially Protein have greater satiety-enhancing potential than fat [27,38-41] and the results of this study support this observation. However, the macronutrient-dependent response on satiety become weaker across the measurement period, so that finally satiety scale values noted $240 \mathrm{~min}$ postprandial are all within a similar range (control excluded). Further investigations would be required to determine the exact mechanism(s) by which Protein and carbohydrates enhance satiety. However, there is evidence in the literature which indicates that macronutrients that induce a high thermic effect may lead to an increased suppression of hunger $[33,42,43]$.

Moreover, these findings agree with the suggestion given by Jéquier [40] that the small, fat-induced influence on satiety may be explained by the fact that appetite regulation signals, such as Cholecystokinin [CCK], are too weak or occur too late to avoid excessive food intake. Supporting Jéquier's thesis, it was observed that that the Fat-rich breakfast initiated the lowest, and a temporally delayed, satiety feeling, even though all test breakfasts were isoenergetic (control excluded). With regard to healthy weight management, this would be an inappropriate effect.

It was also investigated if the total weight of the meal consumed impacted satiety, as the volume of the stomach content should activate satiety signals. We found that, the higher the total weight (Table 3) of the breakfast variation, the higher the sum of the postprandial satiety scale values (Table 6). Nevertheless, at the end of the morning test ( $240 \mathrm{~min}$ postprandial), no significant differences in satiety scale values were observed. It is possible that there must be further satiety-influencing factors. Finally, to explore and understand the effects of a Protein-rich meal to reduce in-between meal snaking to reduce energy intake or portion size at lunch and influence macronutrient choice in following meals further research is required.

One of the limitations of this study was that external factors, such as the physical and mental states of the subjects or their recent dietary intake may have affected the results. In addition, to offer an acceptable breakfast suitable for daily use, the percentage of Protein energy of the Protein-rich breakfast was lower than the percentages of carbohydrate and fat energy of the Carbohydrate and Fat-rich breakfasts, respectively. The "real" Protein-induced effects were underestimated. Another difficulty in interpreting these results is that macronutrients' source and type have not been taken into account for the preparation of the Carbohydrate, Fat and Protein-rich breakfasts. Furthermore, all postprandial parameters were only followed up over four hours of test meal. The diet-induced effects over the entire day were not assessed and total daily energy intake and macronutrient composition of later meals were not recorded. To improve knowledge of the optimal timing of a higher Protein intake, not just the diet-induced effects after breakfast but also after later meals (lunch and dinner) with an increased Protein-load should be investigated. Moreover, women were not included in the study as we aimed to reduce the effect of hormonal variability, which could have influenced the measured parameters. Finally, all subjects tested were young, fit and of a healthy weight. In view of the fact that changes in age, physical fitness and weight are associated with alterations in metabolic and respiratory parameters, participants who are overweight or obese should be examined in future studies.

\section{Conclusions}

The results of this short-term study suggest that postprandial differences in glucose regulation, energy metabolism and satiety feeling may appear minor when comparing the macronutrient composition of breakfasts. However, the daily contribution of the Protein-initiated combined effects of a reduced glycemic response, an increased postprandial $\mathrm{VO}_{2}$ with a proportionately higher fat oxidation and stronger satiety might be advantageous for weight regulation in healthy weight men. Other studies suggest that the same positive effects could be expected in overweight and obese people. Nevertheless there is still a need for further and long-term studies, to clarify the role of the amount and quality of Protein eaten on a continuing basis and the timing of Protein intake for the prevention and/or treatment of overweight and obesity.

\section{Competing Interests and Disclosures}

Research related of this study was funded by the Almased $\mathrm{GmbH}$, Bienenbüttel, Germany, and A. Berg has written "The Almased Wellness Concept" outlining the effects of a soy-enriched diet in health and disease.

\section{References}

1. Goran MI. Metabolic precursors and effects of obesity in children: a decade of progress, 1990-1999. Am J Clin Nutr. 2001;73(2): 158-71.

2. Biesalski HK, Grimm P. Taschenatlas der Ernährung. 3rd ed. Stuttgart: Thieme-Verlag; 2004

3. Rehner, Daniel. Biochemie der Ernährung. 2nd ed. Berlin: Spektrum Akad Verlag; 2002.

4. Galgani J, Ravussin E. Energy metabolism, fuel selection and body weight regulation. Int J Obes (Lond). 2008; 32 (7): S109-119. doi: 10.1038/ijo.2008.246.

5. WHO (World Health Organisation). The challenge of obesity in the WHO European Region and the strategies for response. 2007; (ISBN $9789289014083)$.

6. RKI (Robert Koch Institut-statistisches Bundesamt). Übergewicht und Adipositas. Gesundheitsberichterstattung des Bundes. Themenheft 2003; 16: 15-18.

7. Berg A, Jakob E, Lehmann M, Dickhuth HH, Huber G, Keul J. [Current aspects of modern ergometry]. Pneumologie. 1990; 44(1): 2-13.

8. Borg GA. Perceived exertion: a note on "history" and methods. Med Sci Sports. 1973; 5(2): 90-3. 
9. Holm S. A Simple Sequentially Rejective Multiple Test Procedure. Scandinavian Journal of Statistics 1979; 6(2): 65-70.

10. Nurul-Fadhilah A, Teo PS, Huybrechts I, Foo LH. Infrequent Breakfast Consumption Is Associated with Higher Body Adiposity and Abdominal Obesity in Malaysian School-Aged Adolescents. PLoS ONE 2013; 8(3): e59297. doi: 10.1371/journal.pone.0059297.

11. Szajewska H, Ruszczynski M. Systematic review demonstrating that breakfast consumption influences body weight outcomes in children and adolescents in Europe. Crit Rev Food Sci Nutr. 2010; 50(2): 113119. doi: $10.1080 / 10408390903467514$.

12. Arora M, Nazar GP, Gupta VK, Perry CL, Reddy KS, Stigler MH Association of breakfast intake with obesity, dietary and physical activity behavior among urban school-aged adolescents in Delhi, India: results of a cross-sectional study. BMC Public Health. 2012; 12 881. doi: 10.1186/1471-2458-12-881.

13. Giovannini M, Verduci E, Scaglioni S, Salvatici E, Bonza M, Riva E, et al Breakfast: a good habit, not a repetitive custom. J Int Med Res. 2008; 36(4): 613-624.

14. Te Morenga L, Mann J. The role of high-protein diets in body weight management and health. Br J Nutr. 2012; 108 (2): S130-138. doi: $10.1017 /$ S0007114512002437.

15.Schusdziarra V, Hausmann M, Wittke C, Mittermeier J, Kellner M, Naumann A, et al. Impact of breakfast on daily energy intake - an analysis of absolute versus relative breakfast calories. Nutr J. 2011; 10: 5. doi: 10.1186/1475-2891-10-5.

16. Purslow LR, Sandhu MS, Forouhi N, Young EH, Luben RN, Welch AA et al. Energy Intake at Breakfast and Weight Change: Prospective Study of 6,764 Middle-aged Men and Women. Am J Epidemiol. 2008; 167(2): 188-192.

17. Spagnoli TD, Bianco L. [Breakfast habits in moderately obese adults and its effect on daily energy and nutrient intake, on alcohol consumption and on various clinical and anthropometric parameters]. Minerva Gastroenterol Dietol. 1995; 41(3): 237-245.

18. Ludwig DS. The glycemic index: Physiological mechanisms relating to obesity, diabetes, and cardiovascular disease. JAMA. 2002; 287(18) 2414-2423.

19. Roberts SB. High-glycemic index foods, hunger, and obesity: is there a connection? Nutr Rev. 2000; 58(6): 163-169.

20. Blaak EE, Antoine JM, Benton D, Björck I, Bozzetto L, Brouns F, et al. Impact of postprandial glycaemia on health and prevention of disease. Obes Rev. 2012; 13(10): 923-984. doi: 10.1111/j.1467789X.2012.01011.x.

21. Balon TW, Welk GJ. Effects of prior exercise on the thermic effect of glucose and fructose. J Appl Physiol. 1991; 70(4): 1463-1468.

22. Galgani J, Ravussin E. Energy metabolism, fuel selection and body weight regulation. Int J Obes (Lond) 2008; 32(7):109-119. doi: 10.1038/ijo.2008.246.

23. Marra M, Scalfi L, Contaldo F, Pasanisi F. Fasting Respiratory Quotient as a Predictor of Long-Term Weight Changes in Non-Obese Women. Annals of Nutrition and Metabolism 2004; 48(3): 189-192.

24. Weyer C, Pratley RE, Salbe AD, Bogardus C, Ravussin E, Tataranni PA. Energy Expenditure, Fat Oxidation, and Body Weight Regulation: A Study of Metabolic Adaptation to Long- Term Weight Change. J Clin Endocrinol Metab. 2000; 85(3): 1087-1094

25.Zurlo F, Lillioja S, Esposito-Del Puente A, Nyomba BL, Raz I, Saad MF, et al. Low ratio of fat to carbohydrate oxidation as predictor of weight gain: study of 24-h RQ. Am J Physiol. 1990; 259 (5 Pt 1): 650-657.

26. Lejeune MP, Westerterp KR, Adam TC, Luscombe-Marsh ND, Westerterp-Plantenga MS. Ghrelin and glucagon-like peptide 1 concentrations, 24-h satiety, and energy and substrate metabolism during a high-protein diet and measured in a respiration chamber. Am J Clin Nutr. 2006; 83(1): 89-94.

27. Halton TL, Hu FB. The Effects of High Protein Diets on Thermogenesis, Satiety and Weight Loss: A Critical Review. J Am Coll Nutr. 2004; 23(5): 373-385.

28. Eisenstein J, Roberts SB, Dallal G, Saltzman E. High-protein Weightloss Diets: Are They Safe and Do They Work? A Review of the Experimental and Epidemiologic Data. Nutrition Reviews. 2002; 60 (7 Pt 1): 189-200.

29. Robinson SM, Jaccard C, Persaud C, Jackson AA, Jequier E. Protein turnover and thermogenesis in response to high-protein and highcarbohydrate feeding in men. Am J Clin Nutr. 1990; 52(1): 72-80.

30. Austin GL, Ogden LG, Hill JO. Trends in carbohydrate, fat, and protein intakes and association with energy intake in normal-weight, overweight, and obese individuals: 1971-2006. Am J Clin Nutr. 2011; 93: 836-843. doi: 10.3945/ajcn.110.000141.

31. Abete I, Astrup A, Martínez JA, Thorsdottir I, Zulet MA. Obesity and the metabolic syndrome: role of different dietary macronutrient distribution patterns and specific nutritional components on weight loss and maintenance. Nutrition Reviews. 2010; 68: 214-231. doi: 10.1111/j.1753-4887.2010.00280.x

32. Larsen TM, Dalskov SM, van Baak M, Jebb SA, Papadaki A, Pfeiffer AF, et al. Diets with High or Low Protein Content and Glycemic Index for Weight-Loss Maintenance. New England Journal of Medicine. 2010; 363(22): 2102-2113. doi: 10.1056/NEJMoa1007137.

33. Westerterp-Plantenga MS. Protein intake and energy balance. Regul Pept. 2008; 149(1-3): 67-69. doi: 10.1016/j.regpep.2007.08.026.

34. Layman DK, Boileau RA, Erickson DJ, Painter JE, Shiue H, Sather C, et al. A Reduced Ratio of Dietary Carbohydrate to Protein Improves Body Composition and Blood Lipid Profiles during Weight Loss in Adult Women. J Nutr. 2003; 133(2): 411-417.

35. Johnston CS, Day CS, Swan PD. Postprandial Thermogenesis Is Increased $100 \%$ on a High-Protein, Low-Fat Diet versus a HighCarbohydrate, Low-Fat Diet in Healthy, Young Women. J Am Coll Nutr. 2002; 21(1): 55-61.

36. Soenen S, Martens EAP, Hochstenbach-Waelen A, Lemmens SG, Westerterp-Plantenga MS. Normal protein intake is required for body weight loss and weight maintenance, and elevated protein intake for additional preservation of resting energy expenditure and fat free mass. J Nutr. 2013; 143(5): 591-596. doi: 10.3945/jn.112.167593.

37. Westerterp KR. Diet induced thermogenesis. Nutr Metab (Lond). 2004; 1(1): 5 .

38. Kushner RF, Doerfler B. Low-carbohydrate, high-protein diets revisited. Curr Opin Gastroenterol. 24: 198-203. doi: 10.1097/ MOG.0b013e3282f43a87.

39. Smeets AJ, Soenen S, Luscombe-Marsh ND, Ueland $\emptyset$, WesterterpPlantenga MS. Energy Expenditure, Satiety, and Plasma Ghrelin, Glucagon-Like Peptide 1, and Peptide Tyrosine-Tyrosine Concentrations following a Single High-Protein Lunch. J Nutr. 2008; 138(4): 698-702.

40. Jéquier E. Pathways to obesity. Int J Obes Relat Metab Disord. 2002; 26 (2): $12-17$. 
41. Stubbs RJ, Van Wyk MC, Johnstone AM, Harbron CG. Breakfasts high in protein, fat or carbohydrate: effect on within-day appetite and energy balance. Eur J Clin Nutr. 1996; 50(7): 409-417.

42. Paddon-Jones D, Westman E, Mattes RD, Wolfe RR, Astrup A,
Westerterp-Plantenga M. Protein, weight management, and satiety. Am J Clin Nutr. 2008; 87(5): 1558-1561.

43. Crovetti R, Porrini M, Santangelo A, Testolin G. The influence of thermic effect of food on satiety. Eur J Clin Nutr. 1198; 52(7): 482-488. 